\title{
Proptosis and a Dermal Lesion as the Presenting Sign of Lung Adenocarcinoma
}

\author{
Carmen Alejandra Porcar Plana ${ }^{a}$ Martín Puzo Bayod ${ }^{b}$ \\ Sergio Santos Alarcón ${ }^{c}$ Jaime Moya Roca ${ }^{a}$ Ezequiel Campos Mollo ${ }^{a}$ \\ ${ }^{a}$ Ophthalmology Department, Hospital Virgen de los Lirios, Alcoy, Spain; ${ }^{b}$ Ophthalmology \\ Department, Hospital Universitario Miguel Servet, Zaragoza, Spain; 'Dermatology \\ Department, Hospital Universitario Doctor Peset, Valencia, Spain
}

\section{Keywords}

Proptosis · Orbital metastases $\cdot$ Lung adenocarcinoma $\cdot$ Dermal lesion

\begin{abstract}
The purpose is to describe a clinical case of orbital metastases as the presentation sign of the primary tumor, and the importance of a multidisciplinary diagnosis. A 70-year-old man attended the Ophthalmology Department referring ocular pain. Mild proptosis and a dermal lesion in the neck were noticed. Biopsy of the dermal lesion and systemic work-up were compatible with lung adenocarcinoma with metastatic dissemination. After one cycle of palliative chemotherapy, patient's medical condition worsened, and he eventually died. Although rare, orbital symptoms can be the initial clinical presentation preceding the diagnosis of the primary silent lung neoplasm. Ophthalmologist has an important role in diagnoses of metastatic orbital cancer. Involvement of the multidisciplinary team is important for diagnosis and treatment.
\end{abstract}

\section{Introduction}

Orbital metastases originate from extra-orbital tumors which involve the orbit by hematogenous dissemination [1]. Metastasis to the orbit occurs rarely (1-3\%) but they are the most common form of malignant orbital tumor.

Only $19 \%$ of orbital metastasis is the presenting feature of systemic malignancy. After systemic workup, in $10 \%$ of cases, the primary tumor remains unknown [1,2]. We report a case with orbital metastases as the presenting sign of lung malignancy and describe the importance of a multidisciplinary management. 
Fig. 1. Skin metastasis in the neck. Skin metastasis presented as a single, $3 \times 2 \mathrm{~cm}$, ulcerated, erythematous nodule at the back of the neck.

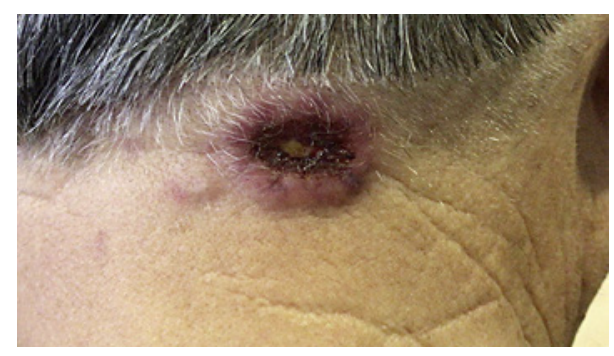

\section{Case Report}

A man in his mid-70s, ex-smoker for 20 years, with a medical history of prostate adenocarcinoma resolved a year ago, was referred to the Ophthalmology Department, presenting left retro-ocular pain and left front hypoesthesia for 1 month. On ocular examination, visual acuity was $20 / 20$ on the right eye and $30 / 40$ on the left eye. Pupillary reaction, confrontational visual field, and ocular motility were normal. Mild exophthalmos with Hertel's exophthalmometer was measured showing $18 \mathrm{~mm}$ right eye and $22 \mathrm{~mm}$ left eye. Hypoesthesia in $V 1$ distribution was noticed with normal sensibility in $V 2$. Slit-lamp examination, intraocular pressure, and fundus exploration were normal.

Expanding anamnesis, patient referred a single, $3 \times 2 \mathrm{~cm}$, ulcerated, erythematous nodule at the back of the neck (Fig. 1). Although the dermal lesion was associated by the patient with a sting, due to its malignancy clinical appearance, the Dermatology Department performed an excisional biopsy. Histopathology examination (Fig. 2) showed a cellular proliferation through the whole dermis thickness, arising to epidermis. Cells were organized in nest with big nuclei and abundant mitosis. The immunohistochemical studies were positive for CK7, TTF1 and showed a granular cytoplasmic staining. p63 and CK 5/6 were negative.

Computed tomographic (CT) scan of the orbit without contrast revealed a lytic mass involving left orbital roof and lateral wall (Fig. 3A), causing exophthalmos (Fig. 3B). Also, 2 hypodense areas in frontal left lobe and left cerebellum were described. Contrast-enhanced brain CT (CeCT) confirmed multiple metastatic nodules in cerebral parenchyma (Fig. 3C).

With all these findings, and a compatible extension CT, lung adenocarcinoma was confirmed as the primary tumor, with a stage IV (T2N2M1) grading. After one cycle of palliative chemotherapy, patient's medical condition worsened, and he eventually died.

\section{Discussion and Conclusions}

Most metastatic cancer to the ocular region occurs in the uveal tract (63\%), followed by the orbit (26\%), and the eyelids (10\%) [1]. As metastasis to the soft tissues of the orbit is relatively uncommon, most ophthalmologist and oncologist have little or none experience with orbital metastasis [2]. The largest series of orbital metastasis reported [2-4] show that orbital metastases occur most frequently (85-68\%) in disseminated disease from an already known primary tumor. Otherwise, orbital metastasis can be the first feature of the primary silent neoplasia (32-9\%). However, in some orbital metastasis cases (17-10\%), the primary tumor remains unknown, despite systemic evaluation and long follow-up.

In all series, primary tumor that most commonly metastasize to the orbit is breast cancer. Although it is variable depending on the study, in most series, lung cancer is the second most common primary cancer, and prostate cancer is the third. In a few instances, the primary cancer is in the gastrointestinal tract, kidney, and skin (melanoma) [2-4].

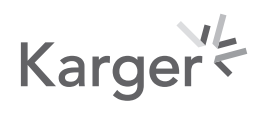




\section{Case Reports in Ophthalmology}

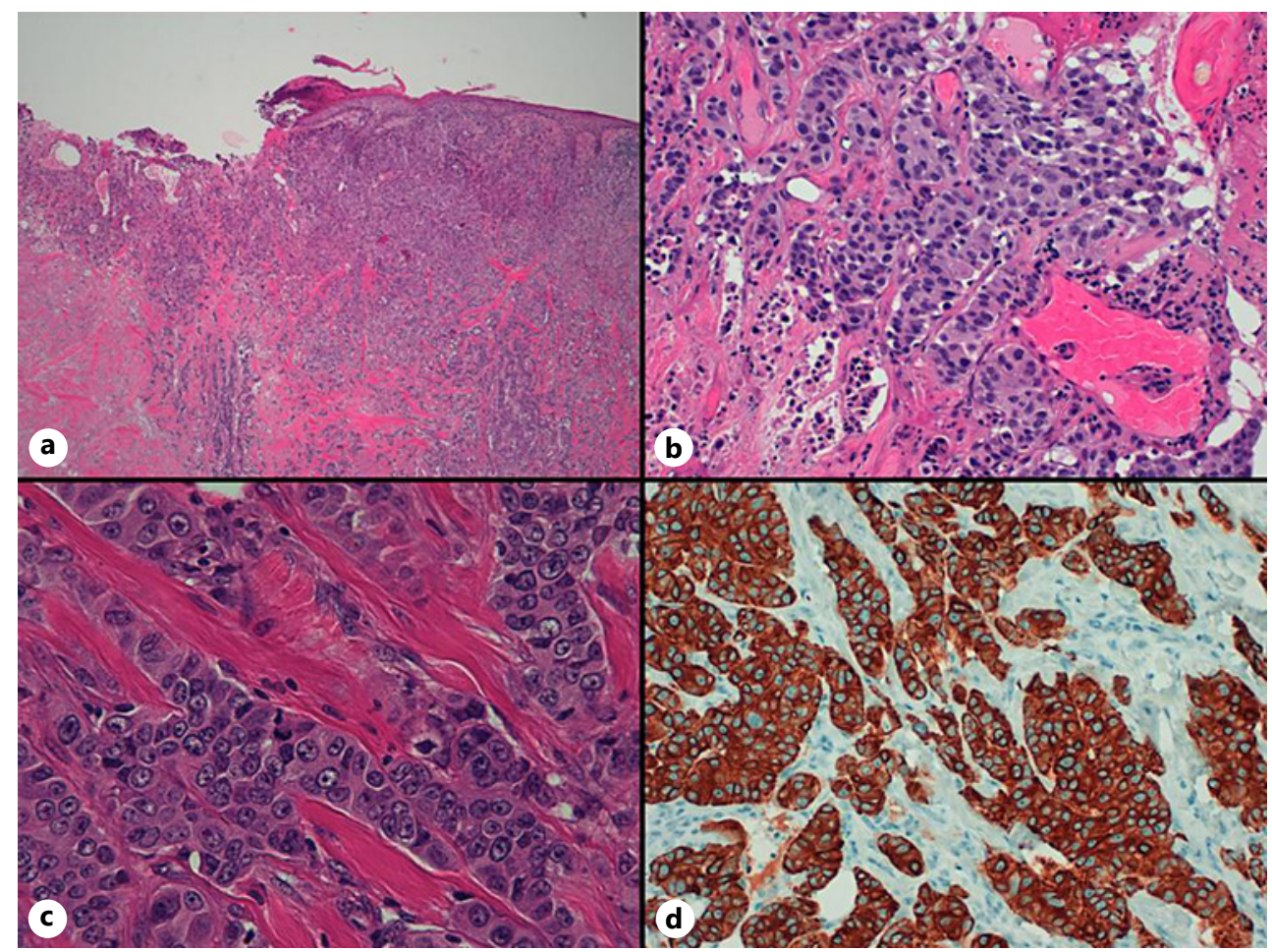

Fig. 2. Histopathology and immunohistochemical examination compatible with lung adenocarcinoma. A Cellular proliferation through the whole dermis thickness arising to epidermis. B Solid cellular growth, organized in nest with wide areas of necrosis. Morphologically (C) there are big cells, with eosinophilic cytoplasm, pleomorphic nuclei, and prominent nucleolus. Mitosis is abundant. Immunohistochemical studies were positive for CK7 (D), TTF1 showed a granular cytoplasmic staining, and p63 and CK 5/6 were negative.
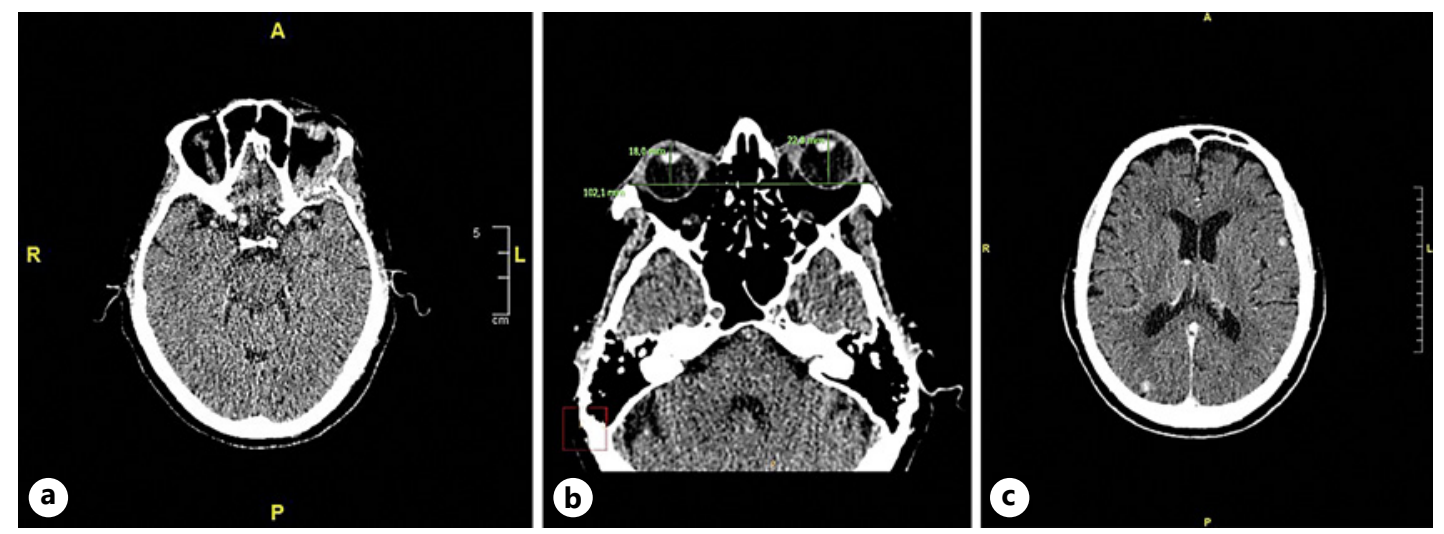

Fig. 3. A, B Orbit computed tomography scan without contrast. C Contrast-enhanced computed tomography scan. A Orbit computed tomography scan without contrast showing osteolytic orbital lesions. B Orbit computed tomography scan without contrast showing exophthalmos measured in millimeters. B Contrast-enhanced computed tomography scan showing brain metastases.

Provisional diagnosis of the primary tumor is a key factor for guiding treatment, as chemotherapy regimens might differ based on primary site [3]. In our patient, a possibility of a relapse of his prostate tumor had to be considered. However, the presence of brain metastases is an anecdotal phenomenon in patients with prostate cancer [5], so an extensive 
work-up study was needed in order to rule out a different primary tumor and establish the disease burden.

As orbital metastasis can be the first feature of a primary tumor, an ophthalmology examination may play a valuable role in diagnosis of cancer. Presentation varies depending on the underlying primary tumor, being diplopia (48\%), pain (42\%), proptosis (26\%), and decreased vision (16-30\%), the most frequent signs and symptoms of orbital metastasis [3]. In this case, proptosis, ocular pain, and incipient decreased vision were probably due to tumor mass effect.

However, the ophthalmology examination must be combined with a complete anamnesis and systemic examination where involvement and cooperation of the multidisciplinary team is important. For example, in our patient finding, a dermal lesion in the systemic evaluation made possible to perform a dermic biopsy which is relatively easy and much more accessible than an orbital biopsy. Orbital metastases generally occur in the late stage of lung carcinoma and are associated with bad prognosis [2], but an early intervention is vital to protect vision and maintain quality of life.

Although rare, orbital symptoms can be the initial clinical presentation preceding the diagnosis of the primary silent lung neoplasm. Ophthalmologist has an important role in diagnoses of metastatic orbital cancer. Involvement of the multidisciplinary team is important for diagnosis and treatment.

\section{Statement of Ethics}

Written informed consent was obtained from the patient for publication of this case report and any accompanying images.

\section{Conflict of Interest Statement}

The authors have no financial disclosures.

\section{Funding Sources}

No funding or grant support.

\section{Author Contributions}

All the authors (Carmen A. Porcar Plana, Martín Puzo Bayod, Sergio Santos Alarcón, Jaime Moya Roca, and Ezequiel Campos Mollo) attest that they meet the current ICMJE criteria for authorship. All of them participated in the clinical management and treatment of the patient, as well as in the elaboration of the paper.

\section{Data Availability Statement}

The authors confirm that the data supporting the findings of this case report are available within the article and its supplementary materials.

\section{Karger's}




\section{References}

1 Campos-Mollo E, Moya-Moya MA, Pérez-González LE, Alenda-González C. [An orbital metastasis as the presentation of lung adenocarcinoma]. Arch Soc Esp Oftalmol. 2005;80(8):467-70.

2 Shields JA, Shields CL, Brotman HK, Carvalho C, Perez N, Eagle RC. Cancer metastatic to the orbit: the 2000 Robert M. Curts lecture. Ophthalmic Plast Reconstr Surg. 2001;17(5):346-54.

3 Valenzuela AA, Archibald CW, Fleming B, Ong L, O’Donnell B, Crompton J J, et al. Orbital metastasis: clinical features, management and outcome. Orbit. 2009;28(2-3):153-9.

4 Magliozzi P, Strianese D, Bonavolontà P, Ferrara M, Ruggiero P, Carandente R, et al. Orbital metastases in Italy. Int J Ophthalmol. 2015;8(5):1018-23.

5 Bhambhvani HP, Greenberg DR, Srinivas S, Hayden Gephart M. Prostate cancer brain metastases: a singleinstitution experience. World Neurosurg. 2020;138:e445-9. 